\title{
LANDAU-KOLMOGOROV TYPE INEQUALITIES FOR CURVES ON RIEMANNIAN MANIFOLDS
}

\author{
IGOR PARASYUK
}

\begin{abstract}
We obtain Landau-Kolmogorov type inequalities for mappings defined on the whole real axis and taking values in Riemannian manifolds. In terms of an auxiliary convex function, we find conditions under which the boundedness of covariant derivative along the curve under consideration ensures the boundedness of the corresponding tangent vector field. We use the square of the distance function as the auxiliary one to establish counterparts of the Landau Hadamard and the Landau-Kolmogorov inequalities where the norms of higher order derivatives of mapping are replaced, respectively, by the Chebyshev radius of curve and the corresponding iterates of covariant derivative along the curve.
\end{abstract}

Mathematics subject classification (2010): 26D10, 26D20, 53C21.

Keywords and phrases: Riemannian manifold, covariant derivative, Landau-Kolmogorov inequality, Chebyshev radius.

\section{REFERENCES}

[1] G. A. Anastassiou, Ostrowski and Landau inequalities for Banach space valued functions, Math. Comput. Modelling 55, 3-4 (2012), 312-329.

[2] G. A. Anastassiou, Intelligent comparisons: analytic inequalities, Springer, Studies in Computational Intelligence 609, Cham, 2016.

[3] Z. Ditzian, Some remarks on inequalities of Landau and Kolmogorov, Aequationes Math. 12, 2-3 (1975), 145-151.

[4] Z. DitZIAN, Remarks, questions and conjectures on Landau-Kolmogorov-type inequalities, Math. Inequal. Appl. 3, 1 (2000), 15-24.

[5] Z. Ditzian, A Kolmogorov-type inequality, Math. Proc. Camb. Philos. Soc. 136, 3 (2004), 657-663.

[6] S. R. FINCH, Mathematical constants, Cambridge University Press, Encyclopedia of Mathematics and its Applications 94, Cambridge, 2003.

[7] D. Gromoll, W. Klingenberg, and W. Meyer, Riemannsche Geometrie im Grossen, SpringerVerlag, Lecture Notes in Mathematics 55, Berlin-New York, 1968.

[8] J. Hadamard, Sur le module maximum d'une fonction et de ses dérivées, C. R. Soc. Math. France, 1914, 68-72.

[9] M. Hayashi And T. Ozawa, On Landau-Kolmogorov inequalities for dissipative operators, Proc. Amer. Math. Soc. 145, 2 (2017), 847-852.

[10] J. Jost, Riemannian geometry and geometric analysis, Springer-Verlag, Universitext, 4th edition, Berlin, 2005.

[11] T. Kato, On an inequality of Hardy, Littlewood, and Pólya, Advances in Math. 7, 3 (1971), 217-218.

[12] A. KolmogorofF, On inequalities between upper bounds of consecutive derivatives of an arbitrary function defined on an infinite interval, Uchenye Zapiski Moskov. Gos. Univ. Matematika 30 (1939), 3-16.

[13] M. K. Kwong AND A. ZETTL, An alternate proof of Kato's inequality, Evolution equations, Lecture Notes in Pure and Appl. Math. 234, 275-279, Dekker, New York, 2003.

[14] E. Landau, Einige Ungleichungen Fur Zweimal Differentiierbare Funktionen, Proc. Lond. Math. Soc. (2), 13, 1 (1914), 43-49. 
[15] D. S. Mitrinović, J. E. PeČARIĆ, AND A. M. FinK, Inequalities involving functions and their integrals and derivatives, Kluwer Academic Publishers Group, Mathematics and its Applications (East European Series) 53, Dordrecht, 1991.

[16] I. PARASYUK, Hyperbolic quasiperiodic solutions of U-monotone systems on Riemannian manifolds, arXiv:1703.04109v1 [math.DS], 2017, 22.

[17] I. PARASYUK, A counterpart of Landau-Hadamard type inequality for manifold-valued mappings, arXiv:1708.01756 [math.CA], 2017, 5.

[18] I. J. Schoenberg, The elementary cases of Landau's problem of inequalities between derivatives, Amer. Math. Monthly 80 (1973), 121-158.

[19] A. Shadrin, The Landau-Kolmogorov inequality revisited, Discrete Contin. Dyn. Syst. 34, 3 (2014), 1183-1210.

[20] Y. XIAO, Landau type inequalities for Banach space valued functions, J. Math. Inequal. 7, 1 (2013), 103-114. 